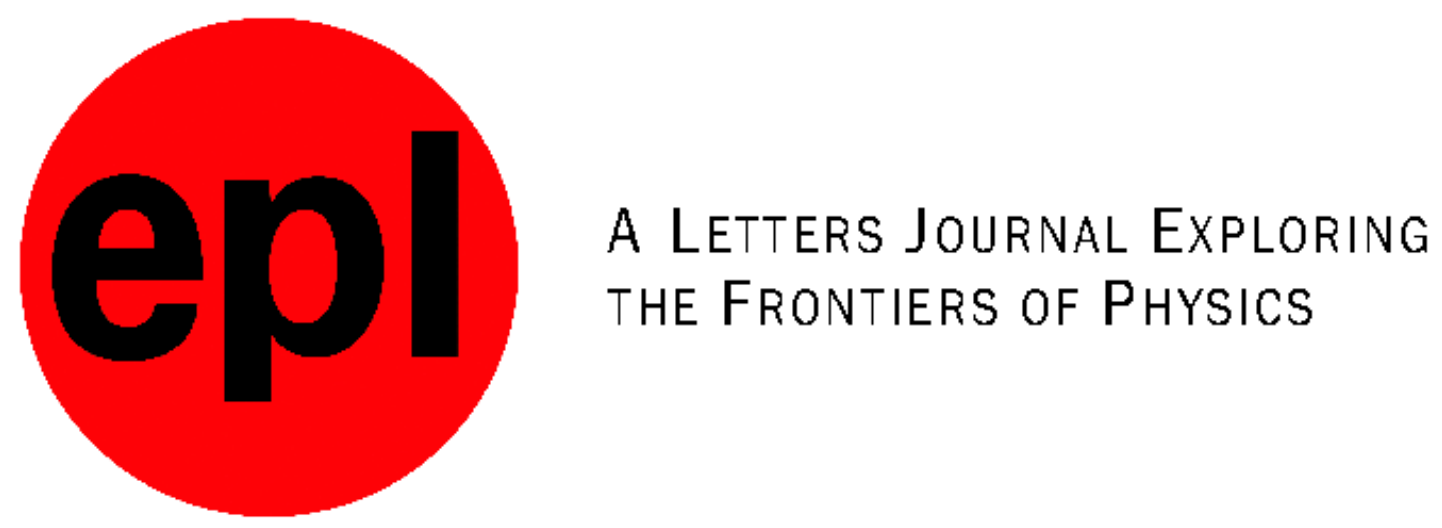

\title{
OFFPRINT
}

\section{Absolute limit for the capillary rise of a fluid}

Frédéric Caupin, Milton W. Cole, Sébastien Balibar and JACQUES TREINER EPL, 82 (2008) 56004

Please visit the new website www.epljournal.org 


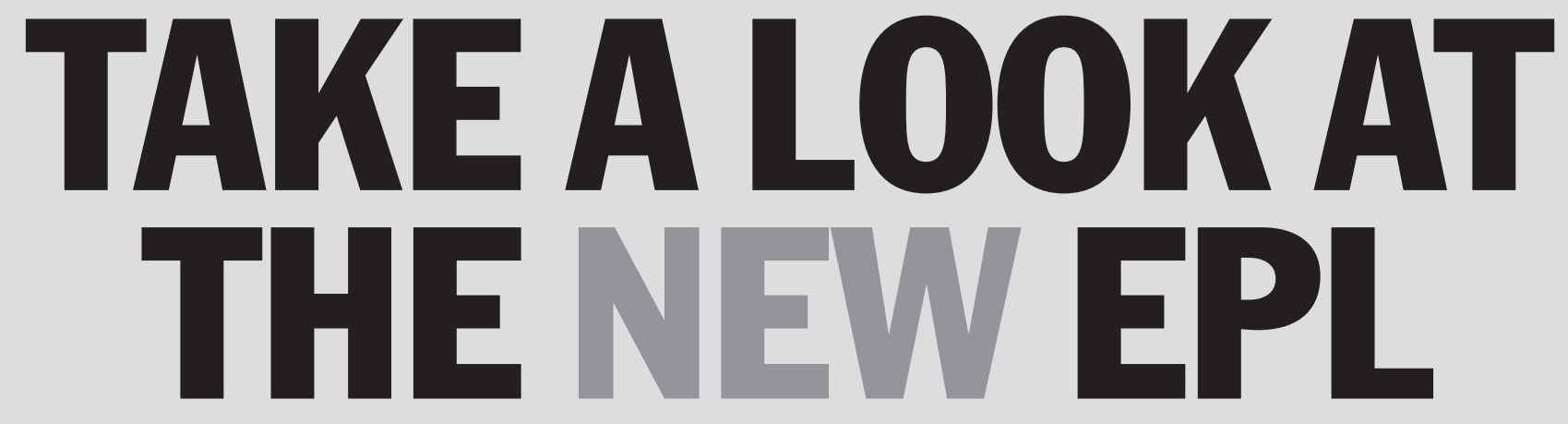

\section{Europhysics Letters (EPL) has a new online home at www.epljournal.org}

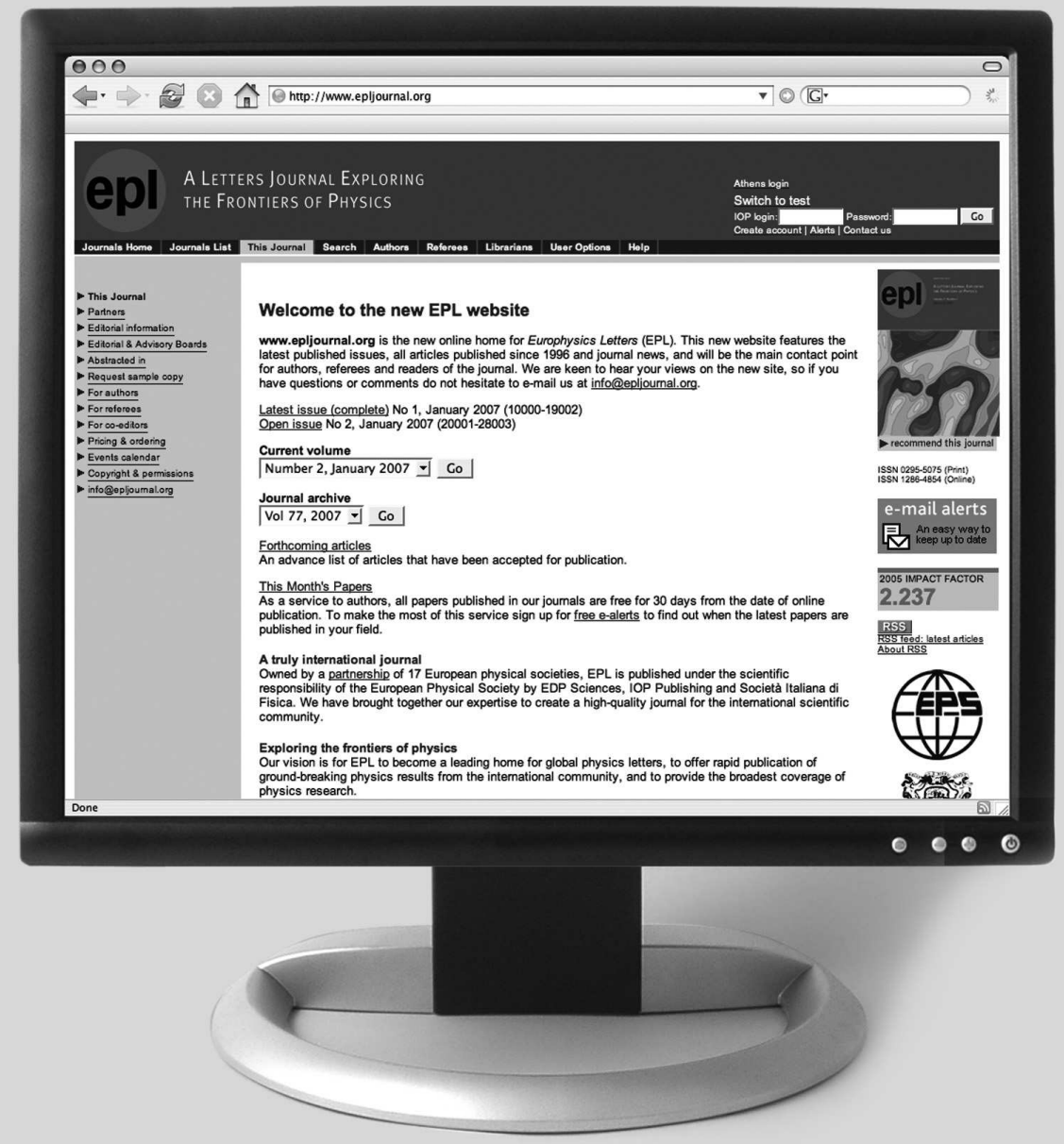

Take a look for the latest journal news and information on:

- reading the latest articles, free!

- receiving free e-mail alerts

- submitting your work to EPL 


\title{
Absolute limit for the capillary rise of a fluid
}

\author{
Frédéric Caupin ${ }^{1(a)}$, Milton W. Cole $^{1,2}$, SÉbastien Balibar $^{1}$ and Jacques Treiner ${ }^{3}$ \\ ${ }^{1}$ Laboratoire de Physique Statistique de l'Ecole Normale Supérieure, associé au CNRS et aux Universités Paris \\ 6 et Paris 7 - 24 rue Lhomond 75005 Paris, France \\ ${ }^{2}$ Physics Department, Pennsylvania State University - University Park, PA 16802, USA \\ ${ }^{3}$ Université Pierre-et-Marie-Curie - 4 Place Jussieu, 75005 Paris, France
}

received 6 November 2007; accepted in final form 14 April 2008

published online 30 May 2008

PACS $67.25 . \mathrm{bh}$ - Films and restricted geometries
PACS $68.08 . \mathrm{Bc}$ - Wetting
PACS $68.43 . \mathrm{De}$ - Statistical mechanics of adsorbates

\begin{abstract}
Small capillaries can provide strong binding to fluids confined within them. We analyze this behavior with a simple microscopic theory, considering two geometries, a slit pore and a cylindrical pore. A goal is to achieve the maximum possible capillary rise $(H)$ within each type of pore. The attraction for very small capillaries can result in a large value of $H$, exceeding $100 \mathrm{~km}$ in a number of cases (e.g., hydrogen, methane and water in cylindrical graphitic pores). The specific value of $H$ depends on the details of the pore and the fluid-surface interaction. It is maximized in the case of small cylindrical pores, strong interactions and small adsorbate mass. Explicit calculations are presented for graphite and $\mathrm{MgO}$ substrates. Experimental tests are possible with an ultracentrifuge, where the high effective gravitational field reduces $H$.
\end{abstract}

Copyright (C) EPLA, 2008

Introduction. - The traditional derivation of capillary rise $(H)$ in a cylindrical tube (radius $R$ ) evaluates $H$ using macroscopic theory and parameters. In the case of a tube that is wet by the fluid, these are the liquid-vapor surface tension $(\gamma)$ and the mass density $(\rho)$ of the fluid. This treatment results in the venerable Jurin's law [1]:

$$
H_{\text {macro }}=\frac{2 \gamma}{\rho g R},
$$

where $g=9.81 \mathrm{~ms}^{-2}$ is the gravitational acceleration.

This equation predicts the capillary rise to be of order a centimeter for typical experiments $(R \simeq 1 \mathrm{~mm})$ used to determine the value of $\gamma$. A curious student might ask whether this relation makes sense when the value of $R$ is of atomic dimensions. For example, the formula yields $H_{\text {macro }} \simeq 30 \mathrm{~km}$ for the case of water at $20^{\circ} \mathrm{C}$ and $R=$ $0.5 \mathrm{~nm}$. Such a value of $H$ seems both immeasurably large and unreliable, since the derivation applies macroscopic laws outside of their regime of validity. Moreover, at a height of $30 \mathrm{~km}$, the pressure of the hypothetical water would be about twice the cavitation limit of water [2], $P_{\text {cav }} \simeq-140 \mathrm{MPa}$, so the water would be ultra-unstable. Nevertheless, motivated by the student's question, one can

(a) E-mail: caupin@lps.ens.fr ask a related question: using more accurate, microscopic physics, what is the maximum possible value of $H$ for a specified fluid?

This paper addresses this latter question. Because the problem, at present, seems "academic", we employ a number of simplifying assumptions which yield semiquantitatively reliable conclusions. The most drastic of these is the assumption that one can construct a regular capillary tube of arbitrarily small $R$, with a value optimized for the specific fluid. A more specific assumption is that the host material can be treated as a continuum in deriving the adsorption potential. With these approximations, we derive for water a value of $H$ that is even greater than the naïve estimate $H_{\text {macro }} \simeq 30 \mathrm{~km}$. However, the physics entering this calculation is rather different; the gas-surface interaction plays a critically important role at the nanoscale, although it plays a relatively minor role (sufficient attraction to achieve complete wetting) at the macroscale.

It has already been seen experimentally that the capillary rise in small (wetting) pores exceeds the prediction from Jurin's law [3]. Such behavior is understood to arise from the fact that a wetting film reduces the effective value of $R$, the radius of curvature of the meniscus [3]. Our treatment is essentially an extrapolation of this physics to the 
limit when the pore space is of atomic dimensions. At the nanoscale, additional information is needed in order to characterize the interaction energies.

We first develop a microscopic description of fluid uptake in slit pores and cylindrical pores, from which the capillary rise is calculated. We then consider the relation between our findings for this problem and for wetting of a flat surface. Finally, we discuss the results and consider experimental methods for testing them.

Analysis. - Traditional analyses yielding the macroscopic expression $H_{\text {macro }}$ use either a force balance or an energy minimization. The energy analysis is equivalent to one used conventionally $[1,4]$ to determine the thickness $d(z)$ of a thick wetting film at height $z$ on a vertical surface, partially immersed in a fluid, which coats the wall when in equilibrium with the bulk fluid. This determination of $d(z)$ is based on the equilibrium condition of uniform chemical potential; it costs no free energy to make a virtual transfer of a molecule from the bulk liquid surface (at $z=0$ ) to the film on the wall. At low temperature one can replace the free energy with the energy, obtaining an implicit expression for $d(z)$ that is used often to characterize thick film adsorption: the so-called "Frenkel-HalseyHill relation" [5]:

$$
M g z+V[d(z)]=0
$$

Here $M$ is the molecular mass and the second term represents the gas-surface potential energy, $V(x)$, provided by the wall to a molecule at the surface of the film, i.e. at distance $x=d(z)$. This relation has been tested experimentally using superfluid helium films, for example; then, a film thickness $d \simeq 10 \mathrm{~nm}$ is observed typically at $z \simeq 10 \mathrm{~mm}$ [5]. See also ref. [6] for a discussion of the maximum height reached by a film climbing along a vertical wall.

This energy analysis is a convenient way to address the present capillary rise problem, thanks to a large body of empirical and theoretical information about adsorption energies. In particular, we determine the maximum height $H$ by finding the most negative energy per particle that can be achieved, considering alternative possible geometries and substrate materials. Let us call $E$ the corresponding optimal binding energy per molecule, while $E_{\text {bulk }}$ is the cohesive energy for the bulk material which coexists, in equilibrium, at the base of the capillary. Then, our working relation analogous to eq. (2) is obtained by substituting $E-E_{\text {bulk }}$ for the potential energy and $H$ for the height:

$$
M g H=E-E_{\mathrm{bulk}} .
$$

For a given attractive gas-surface interaction, we optimize the pore geometry to find the largest value of $E$. In exploring this optimization problem, we have considered simple models, such as pairwise additive potentials (commonly used in modeling adsorption). Such an approach omits many-body interactions, which have been explored but are usually neglected $[5,7,8]$. Nevertheless, the approximations facilitate the study of the many systems we wish to examine and lead to at least qualitative accuracy.

The simplest geometries resulting in large binding of adsorbed fluids are slit pores and cylindrical pores. In discussing these, we make use of two quantities relevant to the interaction $V(x)$ between the molecule and a semiinfinite flat surface. One quantity is $D_{1}$, the well depth of the potential provided by a single surface made of the given material. For the case when one integrates a Lennard-Jones (LJ) 6-12 pair potential over a continuum substrate, of density $n$, the result is [9]

$$
D_{1}=\frac{2 \pi}{9} \sqrt{10} n \epsilon_{\mathrm{gs}} \sigma_{\mathrm{gs}}^{3} .
$$

Here $\epsilon_{\mathrm{gs}}$ is the well depth of the gas-substrate intermolecular pair potential and $\sigma_{\mathrm{gs}}$ is the corresponding "hard-core" parameter. The other quantity is the equilibrium distance for the adsorption potential, $V(x)$; this is $x_{\mathrm{eq}} \simeq 0.86 \sigma_{\mathrm{gs}}$. The assumption of pairwise additivity means that the adsorption potential energies in various geometries are proportional to $D_{1}$, for which experimental data are available; see table 1 .

Considering first the slit pore, one recognizes that the optimal binding occurs when the adsorbed molecules form a two-dimensional (2d) monolayer film, occupying a plane midway between the walls of the materials confining the fluid. Maximum binding occurs when the spacing $L$ between the walls satisfies the relation $L=2 x_{\text {eq }}=$ $1.72 \sigma_{\mathrm{gs}}$. For this choice, the molecules get the maximum attractive energy $\left(D_{1}\right)$ from both adjacent media. Then, the total cohesive energy $E_{\text {slit }}$ includes contributions from the two surfaces $\left(2 D_{1}\right)$, plus the energy $\left(E_{2 \mathrm{~d}}\right)$ per particle from mutual interactions with nearby molecules of the adsorbate:

$$
E_{\text {slit }}=2 D_{1}+E_{2 \mathrm{~d}} \text {. }
$$

The ground-state cohesive energy $E_{2 \mathrm{~d}}$ of a $2 \mathrm{~d}$ LJ solid [10] has been found [7] to have the value $E_{2 \mathrm{~d}}=3.382 \epsilon$, where $\epsilon$ is the well depth of the adsorbate-adsorbate interaction [11]. The ground-state energy of a $3 \mathrm{~d}$ classical LJ solid is $E_{\text {bulk }}=8.6093 \epsilon$ [12]. However, the experimental values [13] give different ratios $E_{\mathrm{bulk}} / \epsilon$ : for instance, they lie between 5.5 and 8 for noble gases. We choose an intermediate value, $E_{\text {bulk }} \simeq 6.7 \epsilon$, bearing in mind the uncertainty due to this choice. We obtain from eq. (3) the height for the slit-pore geometry:

$$
H_{\text {slit }}=\frac{2 D_{1}+E_{2 \mathrm{~d}}-E_{\mathrm{bulk}}}{M g} \simeq \frac{2 D_{1}-3.3 \epsilon}{M g} .
$$

One observes that a large value of $H_{\text {slit }}$ occurs when the substrate attraction greatly exceeds the intermolecular interaction energy.

The analogous optimization problem can be addressed in the case of a cylindrical pore, for which the maximum adsorption potential occurs [9] within a pore of radius 
Table 1: Values of the parameters discussed in the text for various adsorbates in cylindrical and slit pores, listed in order of decreasing $H_{\text {cyl }}$ (also, decreasing $H_{\text {slit }}$ ) for graphite and $\mathrm{MgO}$, as indicated. The decrease of the gravitational force with height (described in the text) has not been taken into account. $\epsilon$ and $\sigma_{\mathrm{gg}}$ values are taken from Berry et al. [14], except for water, in which case the "corresponding states" relations $\epsilon=k_{\mathrm{B}} T_{\mathrm{c}} / 1.313$ and $n_{\mathrm{c}}=0.317 / \sigma_{\mathrm{gg}}^{3}$ were used, where $T_{\mathrm{c}}$ and $n_{\mathrm{c}}$ are the critical temperature and critical point number density, respectively [15]. $D_{1}$ values are taken from Vidali et al. [16]. $\sigma_{\mathrm{gs}}$ values (used to compute $R_{\mathrm{cyl}}$ and $L$ ) are from table 2.1 of ref. [7], where provided, or else from a combining rule: $\sigma_{\mathrm{gs}}=\left(\sigma_{\mathrm{gg}}+\sigma_{\mathrm{CC}}\right) / 2$, where $\sigma_{\mathrm{CC}}=0.34 \mathrm{~nm}$. Quantum corrections for $H_{\text {cyl }}$ and $H_{\text {slit }}$, not included, are significant for ${ }^{4} \mathrm{He}$ and $\mathrm{H}_{2}$, as described in the text.

\begin{tabular}{|c|c|c|c|c|c|c|c|c|c|c|c|}
\hline \multirow[b]{2}{*}{ Substance } & \multirow[b]{2}{*}{$\begin{array}{c}M \\
(\mathrm{amu})\end{array}$} & \multirow[b]{2}{*}{$\begin{array}{c}\epsilon \\
(\mathrm{meV})\end{array}$} & \multirow[b]{2}{*}{$\begin{array}{c}\sigma_{\mathrm{gg}} \\
(\mathrm{nm})\end{array}$} & \multicolumn{5}{|c|}{ Graphite } & \multicolumn{3}{|c|}{$\mathrm{MgO}$} \\
\hline & & & & $\begin{array}{c}D_{1} \\
(\mathrm{meV})\end{array}$ & $\begin{array}{l}H_{\text {cyl }} \\
(\mathrm{km})\end{array}$ & $\begin{array}{l}H_{\text {slit }} \\
(\mathrm{km})\end{array}$ & $\begin{array}{l}R_{\mathrm{cyl}} \\
(\mathrm{nm})\end{array}$ & $\begin{array}{c}L \\
(\mathrm{~nm})\end{array}$ & $\begin{array}{c}D_{1} \\
(\mathrm{meV})\end{array}$ & $\begin{array}{l}H_{\text {cyl }} \\
(\mathrm{km})\end{array}$ & $\begin{array}{c}H_{\text {slit }} \\
(\mathrm{km})\end{array}$ \\
\hline$\overline{\mathrm{H}_{2}}$ & 2 & 3.0 & 0.293 & 52 & 857 & 463 & 0.277 & 0.511 & 48 & 785 & 423 \\
\hline $\mathrm{CH}_{4}$ & 16 & 13 & 0.382 & 130 & 249 & 133 & 0.336 & 0.621 & 135 & 260 & 140 \\
\hline $\mathrm{H}_{2} \mathrm{O}$ & 18 & 42.5 & 0.309 & 161 & 191 & 99.3 & 0.302 & 0.558 & & & \\
\hline${ }^{4} \mathrm{He}$ & 4 & 0.95 & 0.256 & 17 & 140 & 75.9 & 0.255 & 0.471 & 7.5 & 54.5 & 29.2 \\
\hline $\mathrm{N}_{2}$ & 28 & 8.2 & 0.370 & 104 & 118 & 63.6 & 0.331 & 0.611 & & & \\
\hline $\mathrm{O}_{2}$ & 32 & 10 & 0.358 & 102 & 97.8 & 52.6 & 0.325 & 0.600 & & & \\
\hline Ar & 40 & 10 & 0.341 & 96 & 72.8 & 39.1 & 0.317 & 0.585 & 72 & 51.1 & 27.3 \\
\hline $\mathrm{Ne}$ & 20 & 3.6 & 0.275 & 33 & 49.6 & 26.6 & 0.280 & 0.516 & 23 & 31.5 & 16.8 \\
\hline $\mathrm{Kr}$ & 84 & 17 & 0.360 & 125 & 42.5 & 22.7 & 0.319 & 0.588 & 95 & 29.6 & 15.7 \\
\hline $\mathrm{Xe}$ & 131 & 24 & 0.410 & 162 & 34.5 & 18.4 & 0.317 & 0.585 & 121 & 23.2 & 12.2 \\
\hline
\end{tabular}

$R=0.932 \sigma_{\mathrm{gs}}$. The imbibed phase is a $1 \mathrm{~d}$ "axial phase", a line of particles on the cylinder's axis. The adsorption well depth (i.e., the potential on the axis of the cylinder) for this radius satisfies $\left|V_{\text {axis }}\right|=3.68 D_{1}$, nearly twice the magnitude of the potential energy at the midpoint of the optimized slit pore (because of a higher coordination within a cylindrical pore than within a slit pore). Taking into account the fact that the mutual interaction energy of the axial phase is $1.035 \epsilon$ [17], the total cohesive energy of this phase is $E_{\text {cyl }} \simeq 3.68 D_{1}+1.035 \epsilon$. Then for this cylindrical pore we find the capillary rise from eq. (3):

$$
H_{\mathrm{cyl}} \simeq \frac{3.68 D_{1}-5.7 \epsilon}{M g} .
$$

Comparing eqs. (6) and (7), we find that $H_{\text {cyl }}>H_{\text {slit }}$ if and only if $D_{1} / \epsilon>1.4$.

For physical adsorption, it has been found experimentally that graphite is the most strongly attractive surface [16], so that substrate material is one chosen for the numerical calculations in table 1 . The other material is $\mathrm{MgO}$, which has also been the subject of many adsorption studies. For every tabulated adsorbate and both substrates, we find $D_{1} / \epsilon>5$ (except for water which gives 3.8 ); hence the cylindrical environment always yields the higher capillary rise for these materials. The reason is that these are weakly interacting fluids, with small $\epsilon$, which have a strongly attractive interaction with graphite and $\mathrm{MgO}$. The latter energy dominates the physics, in this case, so the more attractive host (cylindrical pore) is more attractive overall, resulting in a higher capillary rise.

The key ratios determining the rise are $D_{1} / \epsilon$ and $D_{1} / M$. For graphite, $\mathrm{H}_{2}$ has (by far) the largest value of the latter and the second highest value (after $\mathrm{He}$ ) of the former.
Hence, among the various materials in table $1, \mathrm{H}_{2}$ is seen to rise highest, to more than $800 \mathrm{~km}$, on graphite, with $\mathrm{CH}_{4}$ and $\mathrm{H}_{2} \mathrm{O}$ having the second and third highest values of both $H_{\text {cyl }}$ and $H_{\text {slit }}$. These three adsorbate species are all particularly light, which is one reason for their large rises ( $M$ appears in the denominator of eq. (7)). Helium is also light, but its well depth is by far the smallest of the tabulated values, so its capillary rise ranks just fourth in table 1 . Note that the ratios $H_{\text {slit }} / H_{\text {cyl }}$ all fall within the interval $[0.52,0.54]$. The reason is that the ratios of the coefficients of $D_{1}$ and $\epsilon$ in their respective formulae (eqs. (6) and (7)) are 0.54 and 0.58 , respectively.

Not surprisingly, the optimal pore sizes $R_{\text {cyl }}$ are comparable to atomic dimensions; this is expected since then the holding potential is the largest possible multiple of $D_{1}$. From a practical point of view, however, this geometry might be impossible to achieve. Suppose, instead, that the pore is significantly wider than $R_{\mathrm{cyl}}$. Then, the capillary rise would involve a different formula. In a first approximation, the binding energy and the cohesive energy would become $D_{1}$ and $E_{2 \mathrm{~d}}$, respectively, leading to $H_{\mathrm{cyl}}=H_{\text {slit }}=$ $\left(D_{1}-3.3 \epsilon\right) /(M g)$. For $\mathrm{H}_{2} \mathrm{O}$ on graphite, a value of $11.3 \mathrm{~km}$ would result, still an extremely high capillary rise, presenting a significant challenge to someone trying to test the prediction.

We note in passing that the simple expression $\left(H_{\text {macro }}\right.$, eq. (1)) neglecting the substrate attraction tends to significantly underestimate the capillary rise found here. For example, for the case of water in a cylindrical pore, using the optimized value of the radius ( $R_{\text {cyl }}$ in table 1$)$, one obtains for graphite the value $H_{\text {macro }}=44 \mathrm{~km}$, while for ${ }^{4} \mathrm{He}$, the value $H_{\text {macro }}=2.1 \mathrm{~km}$ results. These are factors of 4.3 and 67 , respectively, smaller than those shown in table 1. 


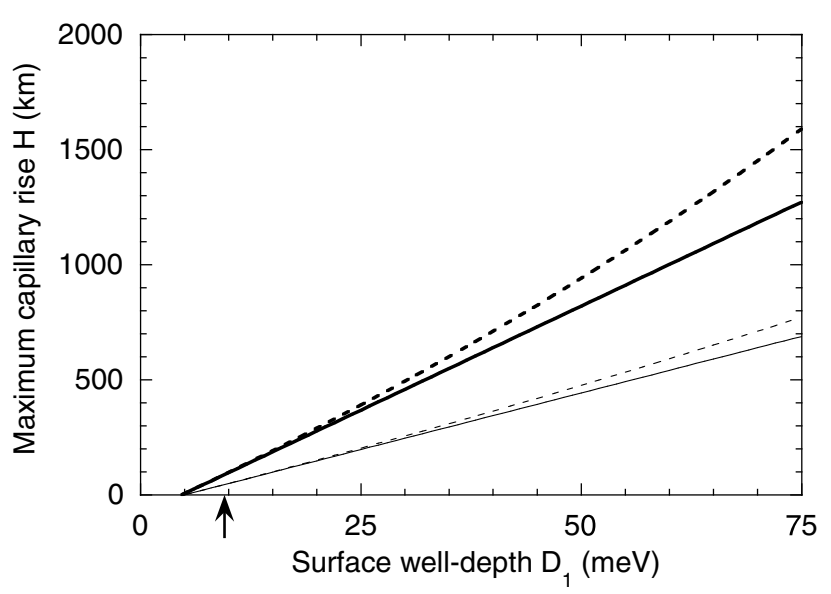

Fig. 1: Capillary rise $H$ of $\mathrm{H}_{2}$ as a function of the well depth $D_{1}$. Bold full (dashed) curve denotes the rise for a cylindrical pore without (with) spherical Earth correction of the gravitational field. Lighter curves represent results for the slit-pore case. The arrow indicates the threshold for wetting on a flat surface (eq. (12)).

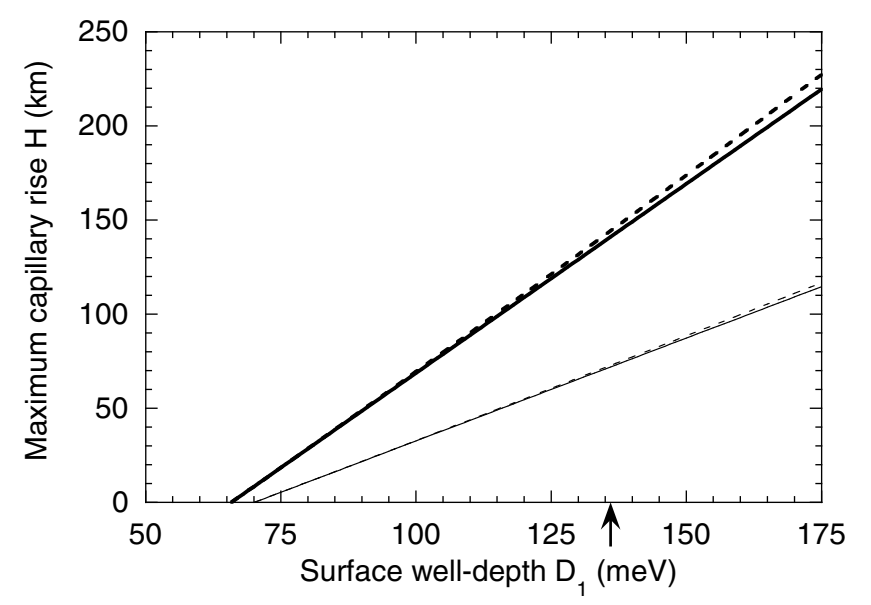

Fig. 2: Same as fig. 1, except for $\mathrm{H}_{2} \mathrm{O}$.

Figures 1 and 2 present the general dependence on well depth of the capillary rise of $\mathrm{H}_{2}$ and $\mathrm{H}_{2} \mathrm{O}$, respectively, in slit and cylindrical pores. The range of plotted values extends only over the values $H>0$. Otherwise, imbibition does not occur. To quantify and discuss this behavior, we define a dimensionless adsorption well depth, $D^{*}=$ $D_{1} / \epsilon$. The resulting threshold criteria are $D_{\text {cyl }}^{*}=1.55$ and $D_{\text {slit }}^{*}=1.65$; below these values no nanocapillary uptake is predicted to occur.

Relation to wetting. - In the Introduction, we discussed the capillary rise for a fluid that (completely) wets the surface, but we did not use that fact explicitly in the calculations at the nanoscale. We may briefly address the relationship between wetting and these latter calculations. The threshold criterion for wetting has been evaluated within the framework of a surprisingly accurate, "simple model", which yields [18]

$$
\left(C_{3} D_{1}^{2}\right)^{1 / 3} \geqslant 3.33 \gamma / n_{\text {liq }}
$$

Here, $n_{\text {liq }}$ is the number density of the liquid and $C_{3}$ is the van der Waals coefficient of the asymptotic gas-surface interaction, $V(x) \sim-C_{3} / x^{3}$; for the case of a sum of LJ pair potentials [7],

$$
C_{3}=\frac{2 \pi}{3} n \epsilon_{\mathrm{gs}} \sigma_{\mathrm{gs}}^{6}
$$

The law of corresponding states provides a scaled value of $\gamma / n_{\text {liq }}$ at the triple point (see table III of ref. [18])

$$
\begin{aligned}
\left(\gamma / n_{\text {liq }}\right)_{\text {triple }} & =\left(\gamma^{*} / n^{*}\right)_{\text {triple }} \epsilon r_{\text {min }} \\
& \simeq 0.83 \epsilon \times 1.12 \sigma_{\mathrm{gg}}=0.93 \epsilon \sigma_{\mathrm{gg}}
\end{aligned}
$$

Here $r_{\min }$ is the equilibrium separation in the pair potential, assumed to have the LJ form. Combining eqs. (4), (8), (9) and (10), we derive an expression for the minimum well depth needed for wetting to occur at the triple point:

$$
\left[D_{1}\right]_{\min }=3.2 \epsilon \sigma_{\mathrm{gg}} / \sigma_{\mathrm{gs}} .
$$

As a very simple approximation, accurate to within $20 \%$ for the tabulated systems, we take the ratio $\sigma_{\mathrm{gg}} / \sigma_{\mathrm{gs}} \simeq 1$, resulting in the wetting criterion

$$
\left[D^{*}\right]_{\min } \simeq 3.2 \text {. }
$$

The corresponding values of $D^{*}$ are thus larger than those derived above $\left(D^{*} \simeq 1.6\right)$ for a significant nanocapillary rise. Thus, the large rise is a necessary concomitant of wetting, as one might expect. However, a large rise can occur even in the absence of wetting (if $1.6<D^{*}<3.2$ ). Thus, the well depth threshold for wetting on a flat surface is more stringent than that of nanocapillary imbibition. This is a consequence of the enhanced substrate attraction in the pore compared to the single planar surface. An example of this behavior is the case of water. While water does not wet graphite at room temperature [19,20], it does strongly adsorb in carbon nanotubes [21,22]. The reasons are the higher coordination possible in the tube than on a flat surface.

Discussion. - Using a straightforward model, we have found that simple fluids should rise to remarkable heights, due to their strong attractions to graphitic materials and $\mathrm{MgO}$. A key assumption used to create table 1 is conventional in physical adsorption: the neglect of manybody interactions. Confidence in the adequacy of this assumption is based on experience with related substrates (graphite, nanotubes and fullerenes) made of carbon. For these, many-body interactions play a relatively small role - typically $15 \%$ or less [23]. In contrast, another assumption, the neglect of quantum effects, is questionable for both $\mathrm{H}_{2}$ and $\mathrm{He}$, for which gases more refined analyses are possible. For $\mathrm{H}_{2}$, based on the quantum calculation 
of Kim et al. [9], one has $E_{\text {slit }} \simeq 100 \mathrm{meV}$, with $E_{\text {bulk }} \simeq$ $7 \mathrm{meV}$, so the resulting rise on graphite would be $H_{\text {slit }} \simeq$ $480 \mathrm{~km}$, much smaller than the tabulated value derived classically. A third assumption is the use of simple LJ interactions; this is particularly problematic for water, for which long-range electrostatic interactions play an important role [24]: as discussed by Zhao [20], these interactions play a different role in adsorption, so that the use of the present model for the capillary rise is more problematic than for other fluids. Finally, we assumed that the gravitational force is $M g$, even at heights of $800 \mathrm{~km}$. In fact, at this height the force is $\simeq 25 \%$ smaller than $M g$. The "corrected" value of the rise, $H^{\prime}$, is given by this equation: $H^{\prime} / H=1 /\left(1-H / R_{\text {Earth }}\right)$, where $R_{\text {Earth }}=$ $6373 \mathrm{~km}$ is the Earth's radius. This expression is valid only when $H<R_{\text {Earth }}$; otherwise, the capillary rise extends to infinity! For $\mathrm{H}_{2}$ /graphite, the correction factor $H^{\prime} / H=$ 1.15 for the cylindrical-pore case and 1.08 in the slit-pore case, as seen in fig. 1. Thus, from this correction alone, we find a revised maximum rise of $\left[H_{\mathrm{cyl}}\right]_{\mathrm{rvsd}}=990 \mathrm{~km}$, for $\mathrm{H}_{2}$ within a graphitic medium.

An interesting question to address is how the predicted capillary rise avoids the cavitation instability, which is expected to occur for bulk water when it rises some $30 \mathrm{~km}$ above the Earth's surface. The answer is that negative pressures are avoided completely in the porous environment because the attractive binding within the pore provides a compressional force, opposing gravity. Thus, the equation determining the local pressure gradient, $\nabla P(\mathbf{r})$, which involves the local number density $n(\mathbf{r})$, has two competing terms:

$$
\nabla P(\mathbf{r})=-n(\mathbf{r}) \nabla[M g z+V(\mathbf{r})] .
$$

The presence of the strongly attractive substrate term means that negative pressures are avoided in the capillary; because of the competing forces, the pressure is elevated within the pore (actually becoming a nondiagonal tensor in the anisotropic porous environment). This behavior is commonly found in films on graphite, where the nearestneighbor spacing is reduced below the value in bulk materials. This high-density film exploits the strong substrate attraction, with an enormous $2 \mathrm{~d}$ spreading pressure at monolayer completion.

The present discussion has not addressed the kinetics of filling these pores, which adds to the already daunting problem of constructing an enormous nanocapillary. This issue is not straightforward since relatively little is known about the kinetics of quasi-2d [25] and quasi-1d films. In macroscopic capillaries, the rise is described by the LucasWashburn $(\mathrm{L}-\mathrm{W})$ equation $[1,26]$. This is a complicated expression, in general; neglecting gravity, however, it simplifies to the form

$$
H(t)=\sqrt{\frac{\gamma R t}{2 \eta}},
$$

where $\eta$ is the shear viscosity of the liquid. Both simulations $[27,28]$ and experiments $[29,30]$ have been used recently to investigate fluid uptake dynamics at the nanoscale. The qualitative predictions of the $\mathrm{L}-\mathrm{W}$ equation have been borne out, even for $R \simeq 1 \mathrm{~nm}$ capillaries. Using this equation, we find for water $\left(\eta=10^{-3} \mathrm{Pas}\right.$, $\left.\gamma=72 \mathrm{mN} \mathrm{m}^{-3}, \rho=10^{3} \mathrm{~kg} \mathrm{~m}^{-3}\right)$ and $R=0.332 \mathrm{~nm}$ a characteristic capillary rise time $t_{0} \simeq 5 \cdot 10^{9}$ years. This result is discouraging since it is not amenable to experimental testing.

One may then wonder about the possibility of any experimental observation of the effect considered. We propose to use a centrifuge. Indeed, in such an apparatus, the apparent gravity ( $\left.g_{\text {appar }}\right)$ is increased by a factor $N$, i.e. $g_{\text {appar }}=N g$. The capillary rise varies as $1 / N$, according to the Jurin equation, and the time varies as $1 / N^{2}$. These dependences have been confirmed in centrifuge studies of the capillary rise in soils [31]. To reach experimentally observable values in nanopores, one needs a large value of $N$, corresponding to a high rotational frequency $\Omega$. This can be achieved with an ultracentrifuge. More precisely, let $r_{0}$ and $r$ be the distance to the rotation axis of the fluid reservoir and of the meniscus, respectively. The equivalent height $H$ is then

$$
H=\frac{\left(\Omega r_{0}\right)^{2}}{2 g}\left[1-\left(\frac{r}{r_{0}}\right)^{2}\right]=\frac{N r_{0}}{2}\left[1-\left(\frac{r}{r_{0}}\right)^{2}\right],
$$

where $N=r_{0} \Omega^{2} / g$. With the characteristics of a swingingbucket rotor of Beckman Coulter ${ }^{1}$ (model SW $60 \mathrm{Ti}$, $r_{0}=120.3 \mathrm{~mm}, r=63.1 \mathrm{~mm}$ and $\Omega=6 \cdot 10^{4} \mathrm{rpm}$ ) one finds $N=4.8 \cdot 10^{5}$ and $H=21 \mathrm{~km}$. Considering that a suitable material for such an experiment could be a porous glass, the well depth is smaller than graphite, and the pore size larger than the tabulated value $R_{\text {cyl }}$. Then $H$ would be significantly smaller than its values in table 1 , making the experiment feasible.

As for the time scale, the previously cited value would be divided by a factor $N^{2}=2.3 \cdot 10^{11}$. The time scale would then become of order seconds, especially if the pore radius is larger than $R_{\text {cyl }}$. To give an idea, let us consider the experiment on Vycor glass (average pore radius $5 \mathrm{~nm}$ ) [30]. Water rises by $26.5 \mathrm{~mm}$ in $2.7 \cdot 10^{4} \mathrm{~s}$, which in the ultracentrifuge would become less than a microsecond. However, such a rapid rise would exceed the velocity of sound! Anyhow, we expect that the experimental time would then be simply limited by the time required to accelerate the centrifuge to a high $\Omega$.

Conclusion. - The results described here are remarkable, at first glance, because the magnitude of the predicted capillary rise is so large. After some reflection, however, the result becomes plausible because the driving force for the rise (excess of adhesive binding energy relative to the cohesive energy) is so large compared to the very weak gravitational potential energy. While the testing of these predictions in pores of altitude

\footnotetext{
${ }^{1}$ Beckman Coulter, Inc., 4300 N. Harbor Boulevard, P.O. Box
} 3100, Fullerton, CA 92834-3100 USA. 
approaching $10^{6} \mathrm{~m}$ is unlikely, the use of the centrifuge method should facilitate their assessment. An obvious question is whether this approach is relevant to the venerable question of why sap rises in tall trees [32]. We hope to address this problem in future work.

$$
* * *
$$

We are indebted to M. Moldover and K. Johnson for helpful remarks. MWC is grateful to the National Science Foundation, Grant DMR-0505160, for its support, and to the ENS for its support and hospitality while he was a visitor there. FC and SB acknowledge support from ANR Grant No. 05-BLAN-0084-01 and Grant No. JC05-48942.

\section{REFERENCES}

[1] De Gennes P.-G., Brochard-Wyart F. and Quéré D., Gouttes, bulles, perles et ondes, 2nd edition (Belin, Paris) 2005; Capillarity and Wetting Phenomena: Drops, Bubbles, Pearls, Waves (Springer, New York) 2004.

[2] Caupin F., Phys. Rev. E, 71 (2005) 051605.

[3] Moldover M. and Gammon R., J. Chem. Phys., 80 (1983) 528; De Gennes P.-G., Rev. Mod. Phys., 57 (1985) 827; Evans R., Fundamentals of Inhomogeneous Fluids, edited by Henderson D. (Dekker, New York) 1992.

[4] Landau L. D. and Lifshitz E. M., Statistical Physics (Pergamon, London) 1958, sect. 146.

[5] Dzyaloshinskit I. E., Lifshitz E. M. and Pitaevski L. P., Adv. Phys., 10 (1961) 165; Cheng E. and Cole M. W., Phys. Rev. B, 38 (1988) 987.

[6] Johnny J. F. and De Gennes P.-G., C. R. Acad. Sci. Paris, 299 (1984) 605.

[7] Bruch L. W., Cole M. W. and Zaremba E., Physical Adsorption: Forces and Phenomena (Dover, Mineola, NY) 2007.

[8] Schmeits M. and Lucas A. A., Prog. Surf. Sci., 14 (1983) 1; Kostov M. K., Cole M. W., Lewis J. C., DieP P. and Johnson J. K., Chem. Phys. Lett., 332 (2000) 26.

[9] Kim H. Y., Gatica S. M. and Cole M. W., J. Phys. Chem. A, 111 (2007) 12439.

[10] The properties of these adsorbed phases at finite temperature $T$ have been studied exhaustively. See, e.g., DAvis H. T., Statistical Mechanics of Phases, Interfaces and Thin Films (VCH, New York) 1996. However, for simplicity, we focus on $T=0$.

[11] See ref. [7], table 5.2; Bruch L. W., Cohen P. I. and Webi M. B., Surf. Sci., 59 (1976) 1. The approximation of energy additivity, eq. (5), is accurate for classical gases at low $T$, while for quantum gases there are small corrections associated with the zero-point motion perpendicular to the plane; see Annett J. F., Cole M. W., Shaw P. B. and Stratt R. M., J. Low Temp. Phys., 84 (1991) 1.
[12] See, for instance, Schwerdtfeger P., Gaston N., Krawczyk R. P., Tonner R. and Moyano G. E., Phys. Rev. B, 73 (2006) 064112.

[13] See ref. [7], table A3.

[14] Berry R. S., Rice S. A. and Ross J., Physical Chemistry (Oxford University Press, Oxford) 2000, Table 21.13.

[15] Pérez-Pellitero J., Ungerer P., Orkoulas G. and Mackie A. D., J. Chem. Phys., 125 (2006) 054515.

[16] Vidali G., Ihm G., Kim H. Y. and Cole M. W., Surf. Sci. Rep., 12 (1991) 133. One exception to the rule seems to be Ne, for which the adsorption potential may be larger for BN than for graphite. Another is $\mathrm{CH}_{4}$, for which $D_{1}$ is larger on $\mathrm{MgO}$ than on graphite (see table 1).

[17] The optimized $1 \mathrm{~d}$ lattice constant is $a=$ $[2 \zeta(12) / \zeta(6)]^{1 / 6} \sigma_{\mathrm{gg}} \simeq 1.1193 \sigma_{\mathrm{gg}}$, where $\zeta(x)$ is the Riemann zeta function. The corresponding cohesive energy is $E_{1 d}=\left[\zeta(6)^{2} / \zeta(12)\right] \epsilon \simeq 1.0347 \epsilon$. See $\mathrm{BRUCH}$ L. W., Surf. Sci., 585 (2005) 135.

[18] Curtarolo S., Stan G., Bojan M. J., Cole M. W. and Steele W. A., Phys. Rev. E, 61 (2000) 1670; Cheng E. et al., Phys. Rev. B, 48 (1993) 18214.

[19] Gatica S. M., Zhao X., Johnson. J. K. and Cole M. W., J. Phys. Chem. B, 108 (2004) 11704.

[20] Zhaо X., Phys. Rev. B, 76 (2007) 041402.

[21] Kolesnikov A. I. et al., Phys. Rev. Lett., 93 (2004) 035503.

[22] Striolo A., Chialvo A. A., Gubbins K. E. and Cummings P. T., J. Chem. Phys., 122 (2005) 234712.

[23] Bruch L. W., Phase Transitions in Surface Films, edited by Taub H., Torzo G., Lauter H. J. and Fain S. C. jr., 2nd edition (Plenum, New York) 1991, p. 67; KIM H.-Y. and Cole M. W., Surf. Sci., 194 (1988) 257; Phys. Rev. B, 35 (1987) 3990; Lambin Ph., Lucas A. A. and Vigneron J.-P., Phys. Rev. B, 46 (1992) 1794.

[24] Guillot B., Mol. Liq., 101 (2002) 219. Note that water/graphite weakly satisfies the wetting criterion, eq. (12), since $D^{*}=3.8$. However, experiments and more accurate modeling find that water does not wet graphite. A wetting transition is predicted to occur near $505 \mathrm{~K}$ $[19,20]$.

[25] Johnny J. F. and de Gennes P.-G., J. Phys., 47 (1986) 121.

[26] Lucas R., Kolloid Z., 23 (1918) 15; Washburn E. W., Phys. Rev., 17 (1921) 273.

[27] Gelb L. D. and Hopkins A. C., Nano Lett., 2 (2002) 1281.

[28] Dimitrov D. I., Milchev A. and Binder K., Phys. Rev. Lett., 99 (2007) 054501.

[29] Nomura R., Miyashita W., Yoneyama K. and Okuda Y., Phys. Rev. E, 73 (2006) 032601.

[30] Huber P., Knorr K. and Kityk A. V., Mater. Res. Soc. Symp. Proc., 899E (2006) N7; Eur. Phys. J. ST, 141 (2007) 101.

[31] Depountis N. et al., Eng. Geol., 60 (2001) 95.

[32] Cochard H., C. R. Phys., 7 (2006) 1018. 